This item was submitted to Loughborough's Research Repository by the author.

Items in Figshare are protected by copyright, with all rights reserved, unless otherwise indicated.

\title{
Closed-loop extended orthogonal space frequency block coding techniques for OFDM based broadband wireless access systems
}

PLEASE CITE THE PUBLISHED VERSION

\section{PUBLISHER}

(C) IEEE

\section{VERSION}

VoR (Version of Record)

\section{LICENCE}

CC BY-NC-ND 4.0

\section{REPOSITORY RECORD}

Eltayeb, Nasr E.M., Shakiru K. Kassim, and Jonathon Chambers. 2019. "Closed-loop Extended Orthogonal Space Frequency Block Coding Techniques for OFDM Based Broadband Wireless Access Systems". figshare. https://hdl.handle.net/2134/5383. 
This item was submitted to Loughborough's Institutional Repository (https://dspace.lboro.ac.uk/) by the author and is made available under the following Creative Commons Licence conditions.

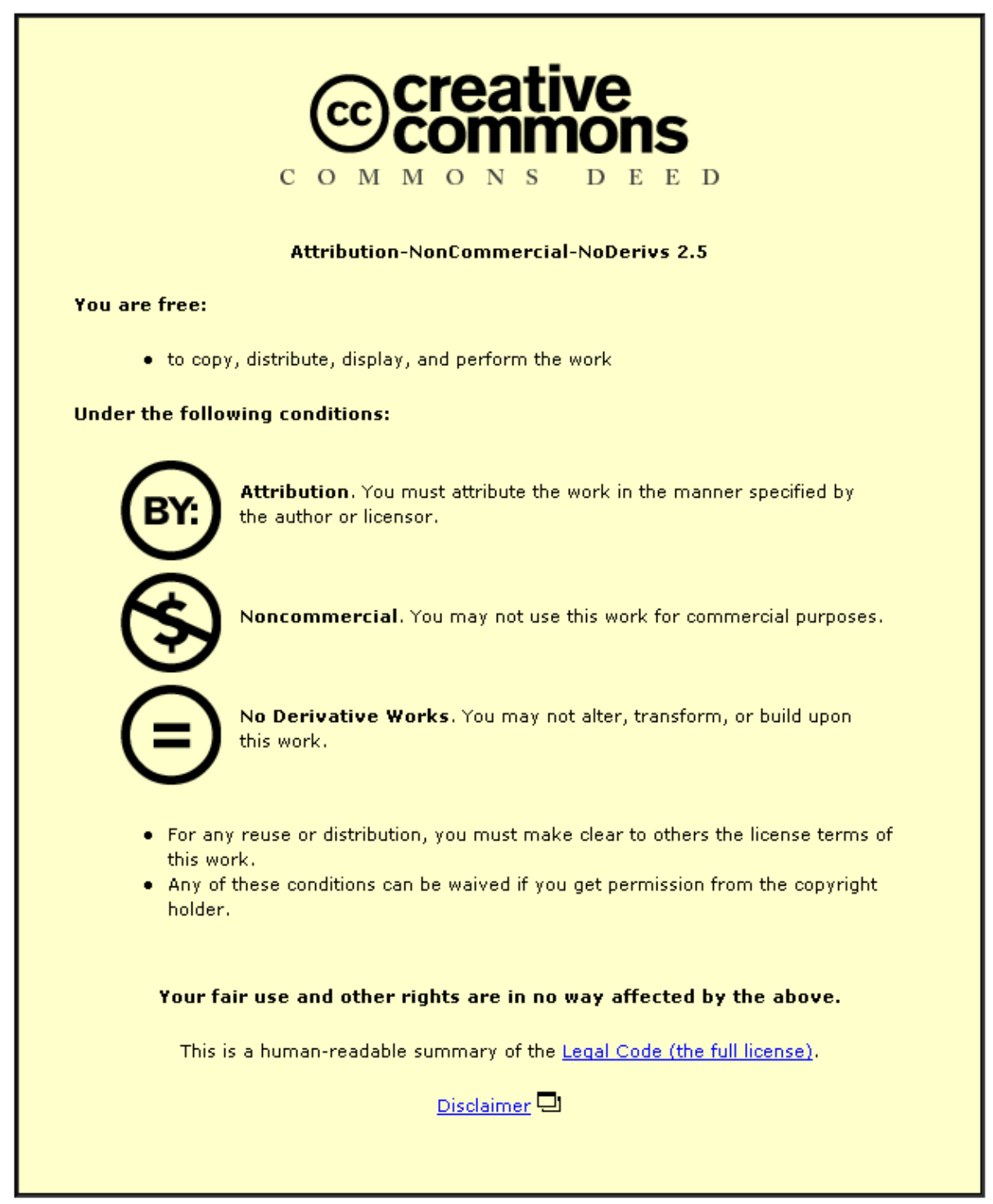

For the full text of this licence, please go to: http://creativecommons.org/licenses/by-nc-nd/2.5/ 


\title{
Closed-Loop Extended Orthogonal Space Frequency Block Coding Techniques for OFDM Based Broadband Wireless Access Systems
}

\author{
N.M. Eltayeb, S.K. Kassim, and J.A. Chambers \\ Advanced Signal Processing Group, \\ Dept. of Electronic and Electrical Engineering, \\ Loughborough University, LE11 3TU, United Kingdom \\ Email: \{n.e.eltayeb, s.k.kassim, j.a.chambers\}@lboro.ac.uk
}

\begin{abstract}
A simple extended orthogonal space-frequency coded multiple input single output (MISO) orthogonal frequency division multiplexing (OFDM) transmitter diversity technique for wireless communications over frequency selective fading channels is presented. The proposed technique utilizes OFDM to transform frequency selective fading channels into multiple flat fading sub-channels on which space-frequency coding is applied. A four-branch transmitter diversity system is implemented without bandwidth expansion and with only one receive antenna. The associated simulations verify that the four-branch transmitter diversity scheme achieves a significant improvement in average biterror rate (BER) performance. The proposed scheme also outperforms the previously reported scheme due to $Y u$, Keroueden, and Yuan with only single phase feedback, and that improvement is retained with quantized feedback. Since the angle feedback is on a per tone basis, the feedback information would be too large for any practical OFDM system. However, we adopt a method which exploits the correlation among the feedback terms for the subcarriers, i.e. a group based quantization technique to reduce the feedback overhead significantly, rendering this scheme attractive to broadband wireless access systems. The performance improvement of convolutionally concatenated space-frequency block coding (CCSBC) schemes is also investigated.
\end{abstract}

Index Terms- Closed-loop (EO-SFBCs), Phase rotation, array gain, diversity gain, coding gain.

\section{INTRODUCTION}

Spatial diversity combined with OFDM is an effective technique for mitigating detrimental effects in broadband wireless multipath fading channels. A key element of OFDM is the utilization of a large number of independent harmonically spaced sub-carriers which are formed in a computationally efficient manner with the fast Fourier transform (FFT). The use of multiple antennas for diversity can enhance channel capacity, making MISO a very attractive practical system. Conventionally, multiple antenna systems have been employed at the receiver side. This increase of receiver hardware can be a major drawback, particularly for portable receivers where physical size and cost are restricted. Transmit diversity has therefore received increased interest, typically for the downlink, as it can achieve diversity gain without increasing the size and complexity of the portable receiver.

Research on adapting several closed-loop methods for STBCs has been proposed to attain full code rate and full diversity. In [1] and [2] the phases of the signals transmitted from certain antennas are rotated in a prescribed way in order to make the code orthogonal based upon the information fed back from the receiver, thereby increasing the diversity gain. Consequently, these techniques are often only effective over slowly-varying flat fading channels.

Space-time coded OFDM (ST-OFDM) systems [3][4], have been proposed for frequency selective fading channels. In [4], it was shown that OFDM modulation with cyclic prefix can be used to transform frequency selective fading channels into multiple flat fading channels, so that orthogonal space-time transmitter diversity can be applied, even for channels with large delay spread. Another approach for transmission over MISO channels using OFDM is to replace the "time" dimension with the "frequency" dimension. The result is called spacefrequency coded OFDM (SF-OFDM) transmitter diversity, which has also been suggested in [3].

In this paper, we propose closed-loop space-frequency block coding (SFBC) algorithms for OFDM to achieve full transmit diversity in terms of transmit antennas and array gain, and demonstrate that one bit feedback per subcarrier is adequate to obtain satisfactory performance. In particular, we develop a new scheme to maximize the signal to noise ratio (SNR) improvement as compared to previously reported work in [2] and [5]. For reference, quasi-orthogonal space-frequency coded OFDM (QO-SFBC) has also been considered [1]. Simulation results show that the average bit error rate performance of the proposed scheme outperforms previous schemes even when the scheme is quantized and when the fading channel is frequency-selective. Moreover, by exploiting feedback correlation among subcarriers, a group based quantization technique is proposed to reduce the feedback overhead significantly, while maintaing satisfactory performance, making this scheme very attractive to broadband wireless access such as WLANs and WMANs such as IEEE 802.11a [6], IEEE 802.16a [7]. In addition, the performance improvement is also investigated when the proposed scheme is combined with convolutional codes, so as to exploit coding gain. Other coding schemes such as turbo-like techniques are not considered due to their larger complexity.

The organization of this paper is as follows. In Section II we describe the closed-loop EO-SFBC for OFDM. In Section III, the concatenation of the closed-loop method 


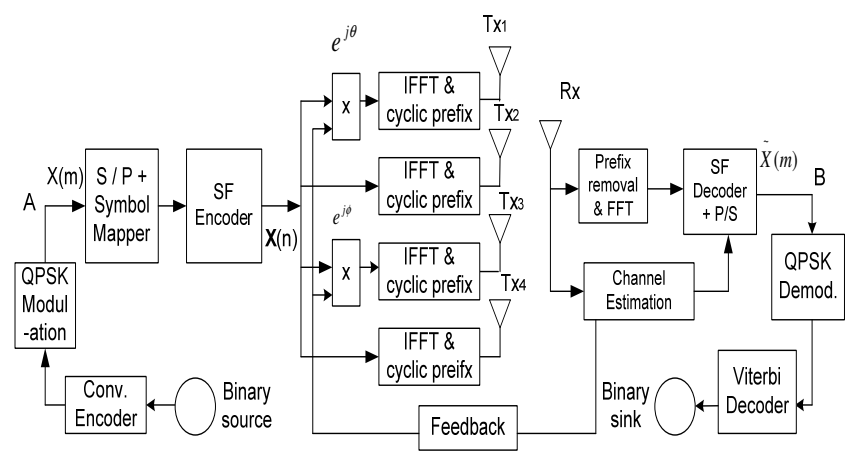

Fig. 1. Baseband representation of the proposed closed-loop space-frequency MISO-OFDM transmitter diversity system.

with convolutional codes (CCSBC) is explained. Simulation results are presented in Section IV and finally some concluding remarks are provided in Section V.

\section{Closed-Loop EO-SFBC For OFDM}

The proposed EO-SFBC scheme for the enhancement of diversity with four transmit antennas for an OFDM system is shown in Fig.1. We consider the following $2 \times 4$ EO-SFBC code matrix similar to the code in [2], where a method was proposed to construct full rate SF codes from full-diversity ST codes [8] via a repetition mapping,

$$
\mathbf{C}=\left[\begin{array}{cccc}
X_{0}(n) & X_{0}(n) & X_{1}(n) & X_{1}(n) \\
-X_{1}^{*}(n) & -X_{1}^{*}(n) & X_{0}^{*}(n) & X_{0}^{*}(n)
\end{array}\right]
$$

where the symbol in the $\mathrm{i}$-th row and $\mathrm{j}$-th column is transmitted through the $\mathrm{j}$-th transmit antennas on the $\mathrm{i}$ th frequency tone during the block instant $n$.

By taking the complex conjugate of the second row for convenience, the equivalent received signal vector for one receive antenna can be obtained as $\mathbf{R}(n)=$ $\left[R_{1}, R_{2}^{*}(n)\right]^{T}$ and the equivalent noise vector becomes $\mathbf{Z}(n)=\left[Z_{0}(n), Z_{1}(n)\right]^{T}$. The result is the matrix equation $\mathbf{R}(n)=\mathbf{H}(n) \mathbf{X}(n)+\mathbf{Z}(n)$, with $\mathbf{X}(n)=$ $\left[X_{0}(n), X_{1}(n)\right]^{T}$, and the matrix $\mathbf{H}(n)$ defined as

$$
\mathbf{H}(n)=\left[\begin{array}{cc}
\Lambda_{0}(n)+\Lambda_{1}(n) & \Lambda_{2}(n)+\Lambda_{3}(n) \\
\Lambda_{2}^{*}(n)+\Lambda_{3}^{*}(n) & -\Lambda_{0}^{*}(n)-\Lambda_{1}^{*}(n)
\end{array}\right]
$$

At the receiver, the matched filtering is performed by premultiplying by $\mathbf{H}^{H}(n)$ in each subcarrier, as follows:

$$
\begin{aligned}
\mathbf{H}^{H} \mathbf{R}(n) & =\mathbf{H}^{H}(n) \mathbf{H}(n) \mathbf{X}(n)+\mathbf{H}^{H}(n) \mathbf{Z}(n) \\
\tilde{\mathbf{R}}(n) & =\Delta(n) \mathbf{X}(n)+\tilde{\mathbf{Z}}(n)
\end{aligned}
$$

where $(\tilde{\cdot})$ denotes a quantity preprocessed by $\mathbf{H}(n)^{H}$. It can be shown that as this an orthogonal block code, all the off-diagonal terms of $\Delta=\mathbf{H}^{H}(n) \mathbf{H}(n)$ will be zero. It is thus reasonable to expect the proposed EOSFBC for OFDM transmit diversity system to achieve full diversity. However, the factor $\beta(n)$ as shown in equation (4) may reduce the performance of the scheme. In order to achieve maximum performance, i.e. full diversity and array gain, we propose a feedback scheme to modify the transmitted signals from certain antennas as shown in Fig. 1 by rotating with an appropriate phase angle to ensure that the $\beta(n)$ term is maximized during the transmission period.

$$
\boldsymbol{\Delta}(n)=\left[\begin{array}{cc}
\alpha(n)+\beta(n) & 0 \\
0 & \alpha(n)+\beta(n)
\end{array}\right]
$$

where $\alpha(n)=\sum_{i=0}^{3}\left|\Lambda_{i}(n)\right|^{2}$ and the factor $\beta(n)=$ $2 \operatorname{Re}\left\{\left(\Lambda_{0}(n) \Lambda_{1}^{*}(n)+\Lambda_{2}(n) \Lambda_{3}^{*}(n)\right)\right\}$. The operator $|\cdot|^{2}$ denotes the modulus squared of a complex number and $\operatorname{Re}\{\cdot\}$ its real part.

\section{A. Phase-Rotation}

Assume that the symbols transmitted from the first and the third antennas in the $\mathrm{n}$-th subcarrier are rotated by phasors $e^{j \theta}$ and $e^{j \phi}$ respectively. It is apparent that this does not change the transmitted power. Since the phase rotation on the transmitted symbols is effectively equivalent to rotating the phases of the corresponding channel coefficients, then the $\beta$ term can be written as:

$$
\grave{\beta}(n)=2 \operatorname{Re}\left\{\left(\Lambda_{0}(n) \Lambda_{1}^{*}(n) e^{j \theta}+\Lambda_{2}(n) \Lambda_{3}^{*}(n) e^{j \phi}\right)\right\}
$$

In this case, by rotating with appropriate phase angles to ensure that $\beta(n)$ is maximized, and correspondingly the signal-to-noise ratio (SNR), therefore $\theta$ and $\phi$ are determined as:

$$
\begin{aligned}
& \theta=-\operatorname{angle}\left(\Lambda_{0}(n) \Lambda_{1}^{*}(n)\right) \\
& \phi=-\operatorname{angle}\left(\Lambda_{2}(n) \Lambda_{3}^{*}(n)\right)
\end{aligned}
$$

where angle $(\cdot)$ denotes the phase angles, in radians, for each complex element.

\section{B. Common Phase-Rotation}

If $\theta=\phi$, a common phasor $e^{j \theta}$ can be used to rotate the transmitted symbols from the first and third antennas and the rotation angle is selected from a range between 0 and $2 \pi$. The new $\ddot{\beta}$ term can be written as:

$$
\grave{\beta}(n)=2 \operatorname{Re}\left\{\left(\Lambda_{0}(n) \Lambda_{1}^{*}(n)+\Lambda_{2}(n) \Lambda_{3}^{*}(n)\right) e^{j \theta}\right\}
$$

In this case, the transmitter needs to have knowledge of a single phase. Therefore, $\theta$ is determined as

$$
\theta=-\operatorname{angle}\left(\Lambda_{0}(n) \Lambda_{1}^{*}(n)+\Lambda_{2}(n) \Lambda_{3}^{*}(n)\right)
$$

However, the gain of this scheme would generally be less.

\section{Quantization}

Due to practical limitations, the number of feedback bits required from the receiver to the transmitter should be kept as small as possible. In the sequel, we provide a method to reduce the number of feedback bits while retaining satisfactory forward link performance. Feeding back the exact value of the phase angle with, for example, fixed or floating point resolution requires very large feedback overhead. In a practical application this may not be possible due to the very limited feedback bandwidth. 
Therefore, the phase angles should be quantized, and then these levels are fed back to the transmitter. Suppose, for each phase angle, if we have two bit feedback, then we can feedback only four phase level angles such that the phase angles are from the set of $\{\Theta, \Phi\} \in \Omega=$ $[0, \pi / 2, \pi$ or $3 \pi / 2]$ then for the first antenna phase adjustment, the discrete feedback information corresponding to the phases may be selected according to

$$
\Theta=\arg \max _{\theta \in \Omega} \operatorname{Re}\left\{\left(\Lambda_{0}(n) \Lambda_{1}^{*}(n)\right) e^{j \theta}\right\}
$$

Similarly, for the third antenna phase adjustment, the phases may be selected according to

$$
\Phi=\arg \max _{\phi \in \Omega} \operatorname{Re}\left\{\left(\Lambda_{2}(n) \Lambda_{3}^{*}(n)\right) e^{j \phi}\right\}
$$

In this case the particular selection giving the largest values of (9) and (10) may be preferable, as it would provide the largest array gain and achieve maximum diversity advantage. This idea can also easily be applied to (8). We now make an observation that when the phase angle is not quantized and when both the first and third antenna signals are rotated using the phase angles, this scheme can be considered as a combined beamformer and SFBC [11], where the pairs of first and second antennas and third and fourth antennas are used as beamformers. Moreover, when the phase angles are quantized to only two levels $(0$ or $\pi)$, this scheme is reduced to the scheme proposed in [2]. Hence, the proposed scheme is expected to perform between the scheme in [2] and combined beamformer and SFBC.

\section{Feedback Reduction Via Sub-channel Correlations}

For frequency division duplex (FDD) based OFDM, however, we require at least $\mathrm{N}$ bits feedback for the phase rotation method. This imposes too much of an overhead constraint on the uplink channel to convey very large amount of feedback information. However, for OFDM based WLANs and WMANs (terminals are likely to remain fixed), the channel variations are small as compared to mobile channels in UMTS, hence phase angles can be fedback less frequently. Nevertheless, techniques that could potentially reduce the amount of feedback will yield significant benefits to the broadband wireless access systems. One possibility to reduce feedback overhead is to exploit the strong correlation in the feedback sequence among the subcarriers [9].

For an arbitrary frequency selective channel of length two, we computed the phase feedback required for a 64 point OFDM symbol and depicted in Fig.2. A two level quantized phase $-\pi / 4$ and $\pi / 4$ is also shown in the same figure. We observe that the required phase feedback is a highly correlated waveform in frequency. We exploit this to perform significant reduction in feedback overheads.

\section{CONCATENATION WITH CONVOLUTIONAL CODES}

In wireless applications, it is favorable to have an outer error-control coding to correct errors that remain after inner codes. In this section, concatenation of the proposed

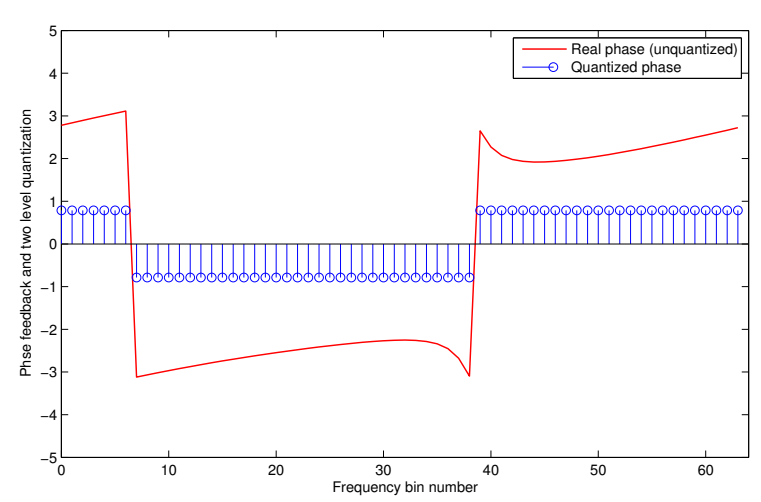

Fig. 2. Quantized and un-quantized phase angles for a 64-point OFDM symbol

scheme with convolutional codes [13] is investigated, due to their low complexity and their practical applicability such as in IEEE $80211 / 16$, as in Fig.1. It is worth noting in channel with bursty errors across frequencies, an interleaver could also be added, however, due to the channel model used in this work this is not included.

Consider that a binary data sequence $s[f], f \in$ $\left\{1, \cdots, L_{c}\right\}$ is to be transmitted. First, it is encoded by the binary convolutional encoder. The output samples of convolutional encoder denoted $c[l], l \in\left\{1, \cdots, N_{c}\right\}$ are mapped onto signal points taken from a QPSK constellation, and the resulting symbols are transmitted according to the EO-SFBC, which is described in Section 2. At the receiver, it is assumed that the CSI is perfectly known. Since the proposed closed-loop EO-SFBCs are decoded using linear processing, the soft values of the symbols of an EO-SFBC codeword can be easily taken from outputs of the matched filter, given in (2). The operation of the convolutional decoder (Viterbi decoder) can be found in many sources (e.g.,[13]), therefore, it is not going to be explained here in detail.

\section{SIMULATION RESULTS}

In this section, we evaluate the error performance of the proposed schemes in quasi-static frequency selective channels. The fading is constant within a frame and changes independently from one frame to another. For the closed-loop system, we have simulated the BER against SNR using QPSK symbols. Each frame consists of 10000 symbols in our simulation. In Fig.3, we provided simulation results comparing the performance of the proposed closed-loop full-rate EO-SFBC for four transmit and one receive antennas. The proposed EO-SFBC with phaserotation feedback obtains better performance than the previous scheme [5], the full rate closed-loop QO-SFBC [9] and the open-loop EO-SFBC respectively. As shown in Fig.3, when the BER is $10^{-3}$, it can yield $1.3 \mathrm{~dB}$ gain over the previous scheme [5], which demonstrates the proposed closed-loop EO-SFBC can get the conventional diversity gain equal to the number of transmit antennas and more additional array gain (feedback gain) at the same time. Furthermore, the performance of the closed-loop QOSFBC with perfect CSI is also depicted, which does not 


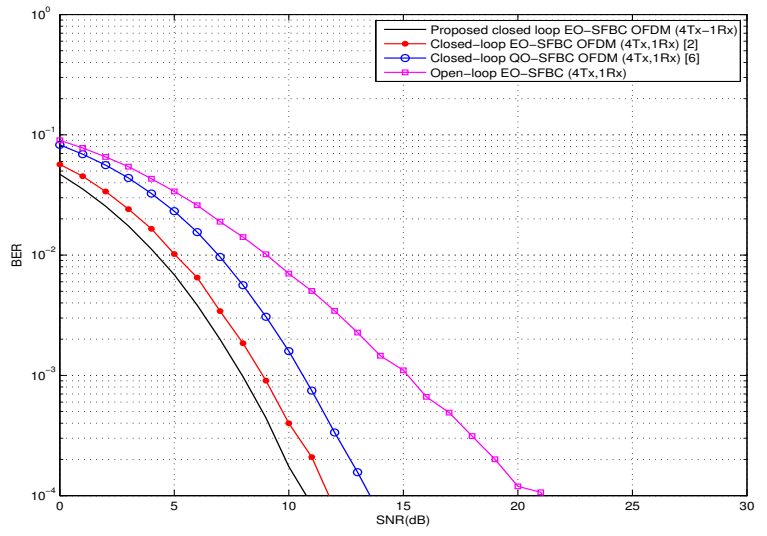

Fig. 3. Average BER performance comparison of the proposed phase rotation scheme with different SFBCs in a $4 \times 1$ MISO system.

benefit from the array gain of the other schemes. The performance of the proposed scheme provides approximately $3 \mathrm{~dB}$ and $7.5 \mathrm{~dB}$ of gain at BER of $10^{-3}$ as compared to QO-SFBC and open-loop EO-SFBC respectively.

The simulation result of a practical scenario of a quantization method is depicted in Fig.4. In addition to this, the un-quantized feedback for the proposed scheme is also depicted. From the figure, simulation results indicate that the performance of the quantized scheme is very close to the performance of the un-quantized scheme, and still significantly better than that of the previously reported closed loop scheme [5]. For the phase rotation method, we quantized the required phase angles into four levels [0, $\pi / 2, \pi, 3 \pi / 2$ ] so that only two bits per subcarrier were required for feedback.

As a tradeoff, we assigned $K$ number of consecutive subcarriers into a group and proposed to feedback one quantized phase angle for each group instead of feeding back the phase angle for each subcarrier. The phase angle required for each group is determined based on the majority of phase angles within that group. In this way, the 64 bit feedback required for one OFDM symbol is reduced to only $M=64 / K$ bits. A result is also shown in Fig.4 for $M=4$. Compared to an un-quantized feedback scheme, where a 64 floating point numbers are used to represent the feedback angles, the quantization with four-bits degrades the BER performance at $10^{-3}$ by only $1.0 \mathrm{~dB}$, but still outperforms closed-loop QO-SFBC and open-loop EO-SFBC by approximately $1.0 \mathrm{~dB}$ and $5.0 \mathrm{~dB}$ respectively.

Although our scheme provides a diversity gain and array gain, an outer channel code can be concatenated to achieve further coding gain. For the final set of simulations, in Fig. 5 we provide performance results when the proposed methods are concatenated with convolutional codes, which is referred to as CCSBC. For this concatenated wireless system, the overall data rate is given by

$$
r=k \log _{2}(m) \quad \text { bits } / \mathrm{sec}
$$

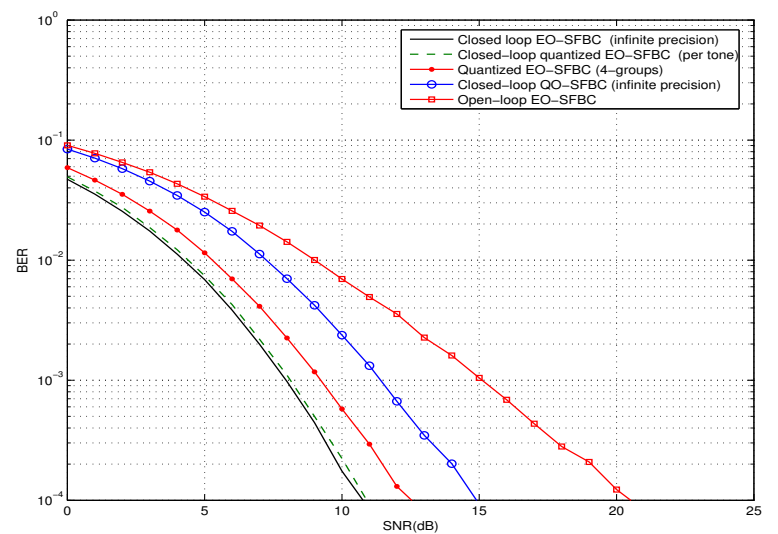

Fig. 4. Average BER performance with quantized and un-quantized phase angles, for $4 \times 1$ system.

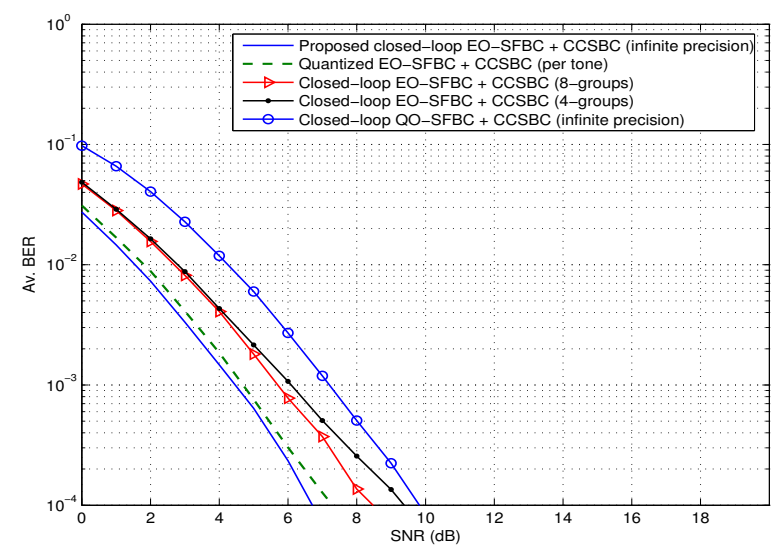

Fig. 5. Average BER performance comparison based on adding outer convolutional code with grouping of feedback terms based quantization, with $M=4$ and 8 .

where $k$ is the code rate of convolutional codes, and $m$ is the dimension of the modulation scheme. In our simulations, we have used a QPSK (i.e. $\mathrm{m}=4$ ) signal constellation with Gray mapping and a rate $1 / 2$ convolutional code (i.e. $k=1 / 2$ ) in full rate closed-loop EO-SFBC systems to achieve the data rate of $1 \mathrm{bits} / \mathrm{sec}$. As depicted in Fig.5, for four transmit antennas, when $\mathrm{BER}=10^{-3}$, CCSBC concatenated with closed-loop EO-SFBC can get $3 \mathrm{~dB}$ from that concatenated with the QO-SFBC code.

\section{CONCLUSIONS}

In this paper, we described an extended orthogonal spacefrequency coded OFDM scheme for providing high datarate wireless communication over frequency selective MISO fading channels. We presented simulation results for a QPSK space-frequency coded OFDM system and compared its performance to the previously reported space-frequency coded OFDM scheme of [5]. We have exploited the feedback correlation among subcarriers to reduce the feedback overheads significantly while 
maintaining satisfactory diversity performance. Furthermore, it is worth noting that the channel matrix of the EOSFBC is orthogonal, which is different from that of the QOSFBC. The proposed closed-loop EO-SFBC scheme with CCSBC can outperform the previous schemes by $4 \mathrm{~dB}$ at BER of $10^{-3}$. This is because in schemes such as CL-QOSFBC there is only diversity gain and no array gain, but in the proposed CL-EOSFBC there is both diversity and array gains. We remark that at each frequency bin that with four rtansmit antennas the maximum diversity for a combined link would be 4 , i.e $N_{t}$. It has been shown in [14] that with the use of linear complex field coding across frequency bins it is possible to obtain maximum diversity of $N_{t} \times(L+1)$, this issue is left as future work.

\section{REFERENCES}

[1] C. Toker, S. Lambotharan, and J. A. Chambers, "Closed-Loop Quasi-Orthogonal STBCs and their Performance in Multipath Fading Environments and When Combined With Turbo Codes," IEEE Trans. Wireless Communications, Vol.3(6), pp.1890-1896, Nov. 2004.

[2] J. Akhtar and D. Gesbert, "Extended Orthogonal Block Codes With Partial Feedback," IEEE Trans. Wireless Commun., Vol.3, no.6, pp.1959-1962, Nov.2004.

[3] D. Agrawal, V. Tarokh, A. Naguib, and N. Seshadri, "Space-Time Coded OFDM for High Data-Rate Wireless Communication Over Wideband Channels," 48th IEEE Vehicular Technology Conference, Vol.3, pp.2232-2236, May 1998.

[4] K. F. Lee, and D .B. Williams, "A Space-Time Coded Transmitter Diversity Technique for Frequency Selective Fading Channels," in Proc. IEEE Sensor Array and Multichannel Signal Processing Workshop, Cambridge, MA, pp.149-152, March 2000.
[5] Y. Yu, S. Keroueden, and J. Yuan, "Closed-Loop Extended Orthogonal Space-Time Block Codes for Three and Four Transmit Antennas," IEEE Signal Processing Letters, Vol.13, No.5, pp.273276, May 2006.

[6] IEEE 802.11a, "High speed physical layer in the $5 \mathrm{GHz}$ band: Wireless LAN medium access control (MAC) and physical layer (PHY) specifications,", 1999.

[7] IEEE 802.16a, "Air interface for fixed broadband wireless access systems: Amendment 2: Medium access control modifications and additional physical layer specifications for 2-11 GHz," 2003.

[8] S. M. Alamouti, "A simple transmit diversity technique for wireless communications," IEEE J. Select. Areas Commun., Vol.16, pp.1451-1458, Oct. 1998.

[9] S. Lambotharan, and C. Toker, "Closed-loop space time block coding techniques for OFDM based broadband wireless access systems," IEEE Trans. Consumer Electronics, Vol.51, pp.765-769, Aug. 2005.

[10] G. Jongren, M. Skoglund, and B. Ottersten, "Combining Beamforming and Orthogonal Space-Time Block Coding," IEEE Trans. Inform. Theory, Vol.48,no.3, pp.611-627, March 2002.

[11] L. Hongwu, and L. Ji, "Space-Frequency OFDM System with Null-Steering Beamformer" ITS Trans. Inf. Theory, pp.341-344, June 2006.

[12] N. Benvenuto, L. Bettella, and R. Marchesani, "Performance of the Viterbi Algorithm for Interleaved Convolutional Codes," IEEE Trans. Vehicular Technology, Vol.47, no.3, pp.919-923, Aug.1998.

[13] N. Benvenuto, L. Bettella, and R. Marchesani, "Performance of the Viterbi Algorithm for Interleaved Convolutional Codes," IEEE Trans. Vehicular Technology, Vol.47, no.3, pp.919-923, Aug.1998.

[14] G. B. Giannakis, Z. Liu, X. Ma, and S. Zhou, Space-Time Coding for Broadband Wireless Communications, John Wiley \& Sons, Inc., January 2007. 